\section{Climate plumbs the depths}

\author{
John Sass
}

IF you wanted to learn about the past climate, your last inclination might be to measure the temperature of soil deep beneath your feet. Yet in the absence of disturbances introduced by convection of groundwater, surface water or local topography, the upper few hundred metres of the Earth store a record of temperature change for the past century or two. This fact is well known to those measuring the Earth's heat flow, for whom the climate signal has been a source of annoyance. But the implications for climate research have not been fully appreciated, which is why a special session was convened at the recent meeting* of the American Geophysical Union.

In the absence of any disturbances, thermal energy from the Earth's deep interior will flow steadily to the surface across a temperature gradient that is a function of the total heat flux (a tiny fraction of the radiant heat from the Sun) and of the thermal resistance of the geological medium. By studying the temperature gradient in boreholes, geophysicists can learn about the heat sources and sinks involved in such geological processes as volcanism, faulting, subduction and basin evolution. But by perturbing the mean surface temperature, climate variations alter the near-surface heat flow. Effectively, the disturbance is propagated down into the crust, leaving a record which can now be recovered (sec figure).

Meteorological obscrvatories are few in number and are mostly located near population centres, sites of surface modification and, consequently, of anthropogenic temperature change. On the other hand, there are many thousands of boreholes worldwide from which equilibrium temperature profiles can be obtained. Where these are close to meteorological observatories, their temperature profiles can be compared with surface temperature records and used to extend records back in time or to recognize emerging trends not apparent in the noisier surface data set. Temperature profiles from locations far from observatories can significantly extend the spatial coverage of the limited observatory data base.

Until recently, research on climatic implications of ground-temperature measurements was concentrated in arctic regions, because of the stability ensured by the thick, impermeable permafrost layer and because the temperature disturbance was larger there during the past century. The focus of the past few years on global warming and the controversy surrounding global climate models and other

*Climate Change Inferred from Borehole Temperature. AGU Fall Meeting, San Francisco, 3-7 December 1990. measures of climate change, have prompted heat-flow researchers to reexamine thermal data from low- and midlatitude boreholes in an effort to identify curvature in the upper parts of temperature profiles and to provide estimates of the size, nature and duration of the surface-temperature disturbances associated with the observed curvature. Heatflow workers have found evidence of warming in the Canadian Shield, the US Great Plains, Cuba, Southern Africa, Antarctica and elsewhere. In some areas, Temperature

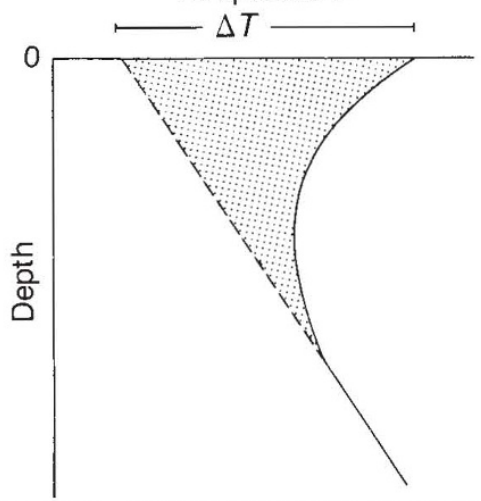

Schematic representation of the effect of surface warming $\Delta T$ on the temperature profile from a borehole (solid line, perturbed profile; broken line, unperturbed). The perturbation typically penetrates $30-40$ metres after a decade, 100-150 metres after a century.

curvature is absent from the temperature profile, or, if present, can be explained in terms of microclimates resulting from nearby lakes or changes in land use. In other areas, there is evidence of cooling, as expected from global climate models.

Although the ground filters out highfrequency variations in atmospheric temperature, ground and air temperatures should follow one another closely over periods of months to decades. Most observatories monitor only air temperatures, so that the complicated heat-exchange process between air and ground has not been well-characterized. Where the two temperatures have been measured, mean annual ground temperatures are usually higher than the air temperatures - by a degree or so in temperate zones, and by several degrees in areas of deep seasonal snow cover

Studies of global air-temperature variations over the past century indicate that the climate has warmed by about $0.5^{\circ} \mathrm{C}$ over that period. This disturbance should be readily apparent in the upper $200 \mathrm{~m}$ of the temperature profile of the Earth and. indeed, many contributors to the special session presented data supporting warming of this magnitude or greater. In general, the temperature history inferred from the borehole measurements in a given region concur with the averaged records of air temperatures from the same region.

Data from Alaskan arctic regions illustrate the great sensitivity of the permafrost layer to surface-temperature perturbations that have occurred over the past century (A. H. Lachenbruch, US Geol. Surv.). The total temperature rise of the Alaskan north slope during the past century is between 2 and $4{ }^{\circ} \mathrm{C}$. Similar increases have been inferred from borehole temperatures in the Canadian Arctic and the Canadian Shield (A. M. Jessop, A. S. Judge, T.J. Lewis, Geol. Surv. Canada; J.-C. Mareschal, H. Beltrami, Univ. Québec). Temperature rises in the range $0.5-2{ }^{\circ} \mathrm{C}$ during the past century were reported from the north-central United States (W. D. Gosnold, M. Bauer, Univ. North Dakota), The Basin and Range Province of Utah (T. J. Chisolm, D. S. Chapman, Univ. Utah), and Southern Africa (H. Pollack, Univ. Michigan).

Although climatic effects can be recognized in temperature profiles (A. E. Beck, Univ. Western Ontario), the inversion of temperature-depth data to extract a measurc of surface temperature as a function of time will not yield an unambiguous thermal history without additional constraints. It is possible, however, to distinguish between, say, a temperature step and a linear increase over time in surface temperature by a careful study of the curvature in the temperature profile. Formal mathematical inversion procedures can be used to establish limits for the resolution of the method as a function of the accuracy of temperature and depth measurements (G. D. Clow, US Geol. Surv.; D. R. MacAyeal, Univ. Chicago).

A cautionary note was sounded (D. D. Blackwell, Southern Methodist Univ.) in that the locations of many boreholes are biased towards sites where the curvature characteristic of surface warming is caused by other factors (as on south-facing slopes and close to bodies of water).

The special session on climate change and borehole temperature provided a lively forum, both for comparing notes among heat-flow specialists, and for dialogue with meteorologists and climatologists. From the data presented, it was clear that surface temperature increases of up to a few degrees during this century are widespread. but not ubiquitous. Theoretical studies provided useful input on the sensitivity and limitations of extracting climate information from temperature profiles. The discussions defined a framework for a systematic study of the thousands of temperature profiles available. We can expect to see important contributions to climate research from this source soon. $\square$

John Sass is at the US Geological Survey, 2255 North Gemini Drive, Flagstaff, Arizona 86001, USA 